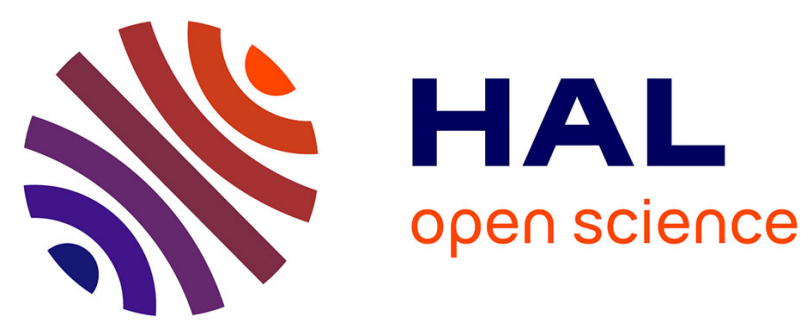

\title{
Superlattice switching from parametric instabilities in a driven-dissipative Bose-Einstein condensate in a cavity
}

Paolo Molignini, Luca Papariello, Axel Lode, R. Chitra

\section{To cite this version:}

Paolo Molignini, Luca Papariello, Axel Lode, R. Chitra. Superlattice switching from parametric instabilities in a driven-dissipative Bose-Einstein condensate in a cavity. Physical Review A, 2018, 98 (5), 10.1103/PhysRevA.98.053620 . hal-02370265

\section{HAL Id: hal-02370265 https://hal.science/hal-02370265}

Submitted on 19 Nov 2019

HAL is a multi-disciplinary open access archive for the deposit and dissemination of scientific research documents, whether they are published or not. The documents may come from teaching and research institutions in France or abroad, or from public or private research centers.
L'archive ouverte pluridisciplinaire HAL, est destinée au dépôt et à la diffusion de documents scientifiques de niveau recherche, publiés ou non, émanant des établissements d'enseignement et de recherche français ou étrangers, des laboratoires publics ou privés. 


\title{
Superlattice switching from parametric instabilities in a driven-dissipative Bose-Einstein condensate in a cavity
}

\author{
Paolo Molignini, ${ }^{1}$ Luca Papariello, ${ }^{1}$ Axel U. J. Lode, ${ }^{2,3,4}$ and R. Chitra ${ }^{1}$ \\ ${ }^{1}$ Institute for Theoretical Physics, ETH Zurich, 8093 Zurich, Switzerland \\ ${ }^{2}$ Wolfgang Pauli Institute c/o Faculty of Mathematics, University of Vienna, Oskar-Morgenstern Platz, 1, 1090 Vienna, Austria \\ ${ }^{3}$ Department of Physics, University of Basel, 4056 Basel, Switzerland \\ ${ }^{4}$ Vienna Center for Quantum Science and Technology, Atominstitut, TU Wien, Stadionallee 2, 1020 Vienna, Austria
}

(Received 24 September 2017; revised manuscript received 27 September 2018; published 20 November 2018)

\begin{abstract}
We numerically obtain the full time-evolution of a parametrically driven-dissipative Bose-Einstein condensate in an optical cavity and investigate the implications of driving for the phase diagram. Beyond the normal and superradiant phases, a third nonequilibrium phase emerges as a many-body parametric resonance. This dynamical phase switches between two symmetry-broken superradiant configurations. The switching is accompanied by a substantial occupation of other momentum states not retained in the mapping to the Dicke model. The emergent phase shows features of nonintegrability and thermalization.
\end{abstract}

DOI: 10.1103/PhysRevA.98.053620

\section{INTRODUCTION}

Quantum light-matter systems present an ideal platform to study the confluence of many-body physics and time-periodic modulations [1,2]. High-frequency modulation is an established versatile tool to experimentally engineer a wide array of static Hamiltonians [3-5]. Near-resonant driving, on the other hand, provides a means of combining unique phenomena like parametric resonance [6], dynamical localization $[7,8]$, and collective many-body physics. Though driven interacting systems tend to heat up [9-11], the interplay between periodic driving and cooperative effects offers the exciting possibility of realizing exotic prethermalized steady states.

Superradiant phases are the quintessential example of collective behavior in light-matter systems [12]. The classic model describing this is the Dicke model [13-16], where a quantum cavity mode collectively couples to independent two-level atoms [14-16]. This physics was recently realized experimentally in a weakly interacting cold bosonic gas coupled to a high finesse optical cavity [17-20], where superradiance manifests itself via the spontaneous formation of a lattice supersolid [21]. Easy implementations of parametric modulations in a wide range of frequencies makes it the perfect realistic system in which to study the influence of drive, interactions as well as dissipation. This is particularly interesting as parametric modulation of the light-matter coupling in the Dicke model (DM) was shown to generate a parametric instability [22,23] to an intriguing new phase of matter, termed the dynamical normal phase (DNP) [23].

In this work, we obtain the phase diagram of a BoseEinstein condensate (BEC) in a dissipative optical cavity with parametrically modulated atom-cavity coupling [see Figs. 1(a) and 1(b)]. Many-body parametric resonance occurs in this system resulting in an emergent oscillatory phase of matter. In this phase, the drive facilitates the dynamical switching of the system between the two symmetry-broken ordered configurations permitted by the undriven Hamiltonian. This switching is, however, explicitly forbidden in the static case. Contrary to standard expectations, this interacting driven-dissipative system displays heating characteristics which depend on its phase, further enriching its physics.

This article is structured as follows. In Sec. II we introduce the model for the coupled BEC-cavity system. In Sec. III we present the results obtained from numerical simulations and analytical calculations. In Sec. III A we reproduce the static phase diagram to benchmark our method and then we obtain and analyze the dynamic phase diagrams for the parametrically driven system in Sec. III B. Section III C is devoted to the analysis of the time evolution of the atomic condensate, while in Sec. IIID we investigate the heating characteristics on the various parametrically driven phases. Last, in Sec. III E we discuss the limitations of the two-level description in the many-body parametric resonance phase (MaPaReP). In Sec. IV we summarize the main findings of our work.

\section{MODEL}

The BEC comprises $N$ interacting atoms and is dispersively coupled to a high-finesse optical cavity with a single mode of frequency $\omega_{c}$, Fig. 1(a). The atoms of the BEC have a transition frequency $\omega_{a}$ and are coherently driven by a transverse pump laser of frequency $\omega_{p}$. In the dispersivecoupling regime, if the atoms are strongly detuned in the rotating frame, i.e., $\Delta_{a}=\omega_{p}-\omega_{a}$ is large, one can adiabatically eliminate the excited atomic levels [24] to obtain the following effective Hamiltonian for the coupled BEC-cavity system in the rotating frame:

$$
\begin{aligned}
\mathcal{H}_{\mathrm{BEC}}= & \int d^{3} r \hat{\Psi}^{\dagger}(\mathbf{r}, t)\left\{-\frac{\hbar^{2}}{2 m} \nabla^{2}+V_{\text {trap }}(\mathbf{r})\right. \\
& \left.+\frac{U}{2} \hat{\Psi}^{\dagger}(\mathbf{r}, t) \hat{\Psi}(\mathbf{r}, t)\right\} \hat{\Psi}(\mathbf{r}, t)
\end{aligned}
$$



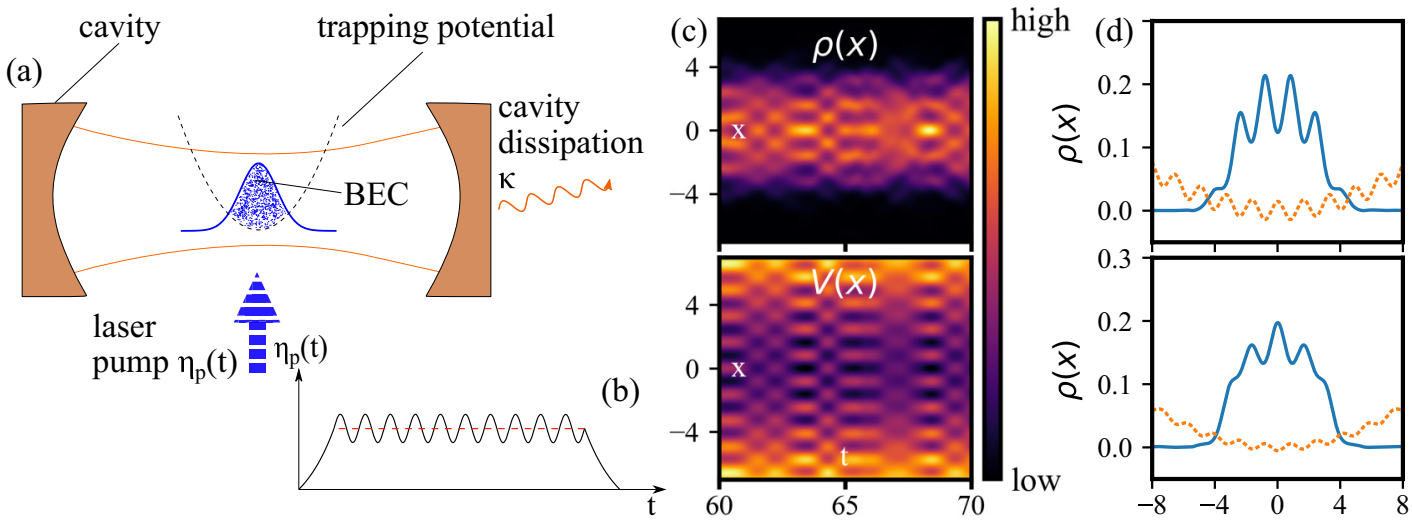

FIG. 1. (a) A BEC in a transversely pumped dissipative cavity, subject to an external harmonic potential of frequency $\omega_{x}$. (b) Laser pump profile $\eta_{p}(t)$, comprising a ramp up, a sinusoidally modulated plateau and a ramp down. (c) Time evolution of the normalized density $\rho(x, t)$ (upper panel) and the self-consistent one-body potential $V(x, t)$ (lower panel) for a time interval of ten units pertaining to the plateau for the many-body parametric resonance phase (MaPaReP). The maximum value reached by $\rho(x, t)$ is 0.296 . (d) Snapshots of the atomic density $\rho(x, t)$ (solid blue line) and one-body potential $V(x, t)$ (dashed orange line) seen by the atoms in the modulated plateau region. In the upper panel, the BEC is mostly localized on the even sites of the periodic lattice. In the lower panel, the BEC is mostly localized on the odd sites. The drive causes atoms of the BEC to oscillate in time between even and odd sites of the periodic lattice in the MaPaReP. A unit of time corresponds to $0.63 \mathrm{~ms}$, while a unit of length corresponds to $0.48 \mu \mathrm{m}$ (see Appendix C).

$$
\begin{aligned}
& +\int d^{3} r \hat{\Psi}^{\dagger}(\mathbf{r}, t)\left\{\frac { \hbar } { \Delta _ { a } } \left[h^{2}(\mathbf{r}, t)+g^{2}(\mathbf{r}) \hat{a}^{\dagger} \hat{a}\right.\right. \\
& \left.\left.+h(\mathbf{r}, t) g(\mathbf{r})\left(\hat{a}+\hat{a}^{\dagger}\right)\right]\right\} \hat{\Psi}(\mathbf{r}, t)-\hbar \Delta_{c} \hat{a}^{\dagger} \hat{a} .
\end{aligned}
$$

The atoms in the BEC are described by bosonic field operators $\hat{\Psi}^{(\dagger)}(\mathbf{r}, t)$ while $\hat{a}^{\dagger}$ and $\hat{a}$ describe the cavity mode. All operators obey bosonic commutation relations. For the sake of computational simplicity, in the following we restrict ourselves to the one-dimensional problem along the cavity axis $x$. The atoms are subjected to a harmonic trapping potential $V_{\text {trap }}(x)=m \omega_{x}^{2} x^{2} / 2$ and interact through shortrange interactions with the strength $U=4 \pi \hbar^{2} a / m$ where $a$ is the $s$-wave scattering length and $m$ is the mass of the atom [25-27].

The atoms are driven by a transverse pump field described by the mode function $h(\mathbf{r}, t)=h(z, t)=\eta_{p}(t) \cos k z$ while the cavity mode function is $g(\mathbf{r})=g(x)=g_{0} \cos k x$. Here $\eta_{p}$ is the pump rate, $k$ is the wavelength of the light, and $g_{0}$ is the atom-cavity coupling. The last two terms in the Hamiltonian describe the atom-cavity interaction. The $g^{2}(\mathbf{r})$ term arises directly from the cavity mode function while the $h(\mathbf{r}, t) g(\mathbf{r})$ term results from the interference between cavity and pump fields.

For static pumps a mean-field analysis for large $N$ and $V_{\text {trap }}(\mathbf{r})=0$ using the Gross-Pitaevskii equation shows a $\mathbb{Z}_{2^{-}}$ symmetry breaking transition; as the pump power increases, the system goes from a normal phase (NP) with no photons in the cavity to a superradiant phase (SP), where the cavity field is a coherent state [17,24,28]. In the SP, the atoms spontaneously self-organize into either an even or an odd lattice structure with a spacing $\lambda=\frac{2 \pi}{k}[17]$. Note that selforganization has been proposed even in the absence of a cavity [29]. The relevant order parameter is $\Theta \equiv\langle\psi|\cos k x| \psi\rangle$ : $\Theta=0$ in the NP and $\Theta \neq 0$ in the SP. $\Theta$ essentially counts the population imbalance between odd and even lattice sites in the SP. The same physics is well described by a mapping to the DM Hamiltonian which assumes that only the lowest $\pm k$ modes of the atoms are populated $[17,28]$.

In this article, we study the parametrically modulated system described by the Hamiltonian (1). The time-dependent pump amplitude in the plateau [see Fig. 1(b)],

$$
\eta_{p}(t)=\eta_{p}^{0}[1+\alpha \sin (2 \pi t / T)],
$$

will lead to a new phase of matter. We explicitly include the cavity dissipation. We consider system parameters which describe the experimental system studied in Refs. [17,18] (see also Appendix C). For the considered system, the cavity dynamics follows that of the atoms closely, because the detuning $\Delta_{a}$ is much larger than the other energy scales. In this adiabatic limit, it is reasonable to replace $\hat{a}$ by its expectation value $\langle\hat{a}\rangle \equiv a$ in the dynamics for the atoms. This cavity population obeys a dissipative equation of motion with a rate $\kappa$ (see Appendix B). We note that, even though semiclassical approaches in the description of dissipation have been shown to capture certain features of collective cooling [15,30], this treatment effectively ignores possible cooling effects induced by cavity fluctuations. The resulting dynamical evolution of the atoms is then studied using the multiconfigurational time-dependent Hartree method for indistinguishable particles (MCTDH-X) [31-34]; see Appendix A for details. This method has been very successful in describing the dynamics of bosonic systems [32-37]. Here we apply the method to a periodically driven many-body system coupled to an optical cavity.

\section{RESULTS}

\section{A. Static phase diagram}

As a benchmark, we reproduce the undriven $\left(\eta_{p}, \Delta_{c}\right)$ phase diagram obtained in [17]. We use the time-dependent transverse pump protocol illustrated in Fig. 1(b) with the modulation amplitude $\alpha=0$ in Eq. (2). At time $t=0$, the 


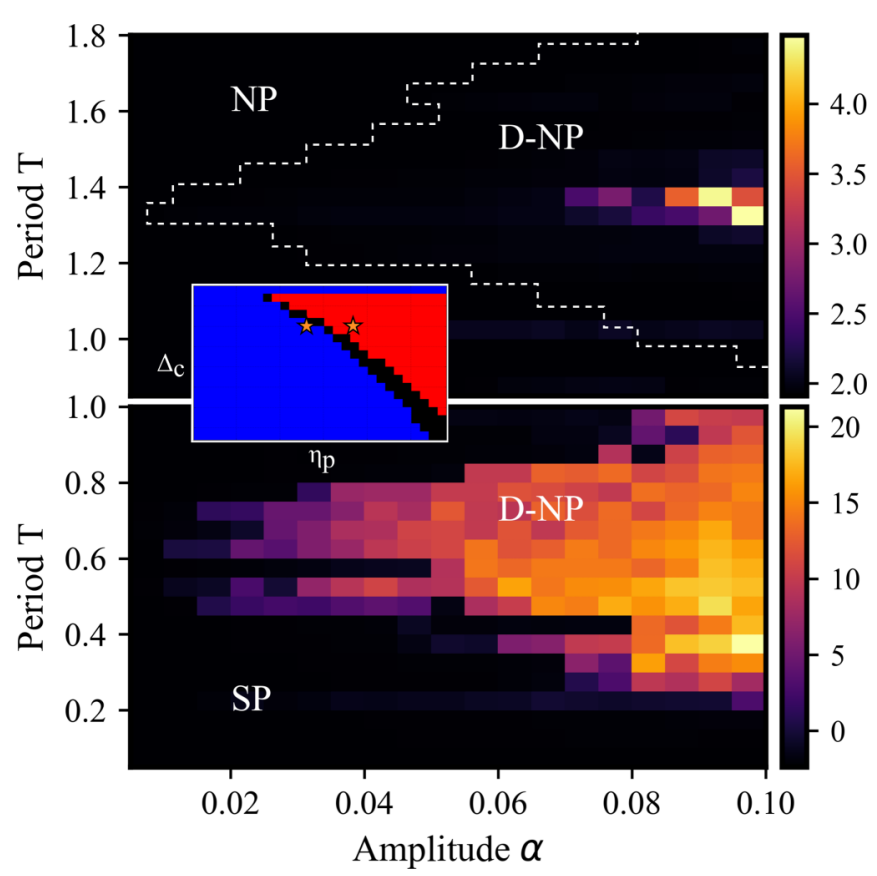

FIG. 2. Phase diagrams of a parametrically driven BEC in a cavity for $N=1000$ bosons. They are superimposed on the average heating of the system as a function of modulation amplitude $\alpha$ and period $T$. The inset shows the undriven phase diagram. The parameters for the driven cases in the NP $\left(\eta_{p}^{0, \mathrm{NP}}=2 \pi \times 3.28 \mathrm{kHz}, \Delta_{c}=\right.$ $-2 \pi \times 10.08 \mathrm{MHz}$; phase diagram in the upper panel) and in the SP $\left(\eta_{p}^{0, \mathrm{SP}}=2 \pi \times 4.79 \mathrm{kHz}, \Delta_{c}=-2 \pi \times 10.08 \mathrm{MHz}\right.$; phase diagram in the lower panel) are marked as stars in the inset. In the upper panel the BEC is driven from the NP and the dashed line, obtained from the evaluation of $\Theta$, delineates the NP and the MaPaReP. In the lower panel the condensate is driven from the SP and the black region corresponds to the SP while the colored region corresponds to the MaPaReP. In both diagrams the Arnold tongue corresponds to the first resonance $(n=1)$ with a period of $T \approx 1.3(\mathrm{NP})$ and $T \approx 0.65$ (SP). In both plots, the black regions indicate no heating while the colored tiles indicate heating. All quantities shown are dimensionless; see Appendix C.

cavity is decoupled from the BEC in the trap. As $\eta_{p}(t)$ is ramped up, and approaches a constant, $\eta_{p}^{0}$, in the plateau we obtain either the NP where $\Theta=0$, or the SP where $\Theta \neq 0$ (see the inset in Fig. 2 and Fig. 7 in the Appendix). For $N=$ 1000 , converged results are obtained using the $M=1$ orbital in the MCTDH-X approach. Contributions from $M>1$ orbitals are negligible. For this $M=1$ case, MCTDH-X corresponds to the mean-field solution obtained using the GrossPitaevskii equation [27]. We verified that our results recover the scaling invariance under $N \rightarrow N^{\prime}$ provided $g_{0} \rightarrow \sqrt{\frac{N}{N^{\prime}}} g_{0}$, $U \rightarrow \frac{N}{N^{\prime}} U$, and $\eta_{p}^{0} \rightarrow \sqrt{\frac{N}{N^{\prime}}} \eta_{p}^{0}[24]$.

\section{B. Parametrically driven system}

We now discuss a nonzero modulation $\alpha$ and assess the nature of the parametrically driven system when it is driven starting either from the NP or from the SP. For a fixed detuning $\Delta_{c}=-2 \pi \times 10.08 \mathrm{MHz}$, we select two representative points close to the SP-NP phase boundary of the static pump simulations (see the inset in Fig. 2). Similarly other pairs of points across the SP-NP phase transition lead to analogous results. Note that $\alpha$ is chosen to be small enough, so that the instantaneous $\eta_{p}(t)$ never crosses the static phase boundaries. In the spirit of standard parametric driving, the modulating frequency $\omega \equiv 2 \pi / T$ is chosen to be close to twice the gap to the lowest polaritonic excitation in the system. The approximate polaritonic gap is determined by mapping the driven BEC-cavity to the DM; see Appendix D. We obtain two polaritonic modes for each phase [38]: a very high-energy branch, $\epsilon_{+}^{\mathrm{NP}-\mathrm{SP}}$ proportional to $\Delta_{c}$, and a low-energy branch $\epsilon_{-}^{\mathrm{NP}-\mathrm{SP}}$ proportional to the atomic recoil energy $2 E_{r}=\frac{\hbar^{2} k^{2}}{m}$,

$$
\begin{aligned}
& \left(\epsilon_{-}^{\mathrm{NP}}\right)^{2} \approx E_{r}^{2}\left[1-\left(\eta_{p}^{0} / \eta_{p, c}\right)^{2}\right], \\
& \left(\epsilon_{-}^{\mathrm{SP}}\right)^{2} \approx E_{r}^{2}\left[\left(\eta_{p}^{0} / \eta_{p, c}\right)^{4}-1\right] .
\end{aligned}
$$

The energy of the lower polaritonic branch goes to zero at the QPT where $\eta_{p} \rightarrow \eta_{p, c}$ (see inset in Fig. 2). To study the impact of parametric driving on the atoms, we simulate the full time evolution of the system as a function of $\omega$ starting from both NP and SP.

Our results for the phase diagram of the modulated BECcavity system as a function of the drive amplitude $\alpha$ and period $T$ are summarized in Fig. 2, obtained by evaluating the order parameter $\Theta$. In the same figure we have also superimposed a color plot of the time averaged energy profile. In the upper panel the phase boundary, obtained from the evaluation of $\Theta$, is indicated with a white dotted line. The energy of the BEC substantially increases only for higher values of $\alpha$. For the lower panel the transition from low- to high-energy zones coincides with the phase boundary obtained from $\Theta$. Both phase diagrams show the emergence of a many-body parametric resonance: the static phases display parametric instability lobes-reminiscent of Arnold lobes for Mathieu oscillators-for certain resonant values of $T[39,40]$. This is related to the fact that in the DM, polaritonic excitations are effectively described by the physics of two coupled parametric oscillators; see Appendix D. For certain thermalizing regions in the phase diagram, however, the mapping to the DM does not fully characterize the physics, as higher momentum states are populated [35] (see also Sec. IIIE). As the parameters $(T, \alpha)$ are varied, the underlying undriven NP or SP become unstable and the system transitions to a new phase which we term the many-body parametric resonance phase (MaPaReP). In this phase, the order parameter $\Theta(t)$ shows oscillatory behavior in time with zero mean (excluding trap contributions).

The instability lobes seen in Fig. 2 differ greatly from the standard Arnold lobes for parametric oscillators described by the Mathieu equation. The periods around which the Arnold lobes of the BEC-cavity system are centered can be calculated as follows. In both phases, the dynamics is essentially governed by a classical Hill equation [39]

$$
\ddot{x}+\gamma \dot{x}+\left[\epsilon_{-}^{\mathrm{NP}-\mathrm{SP}}(t)\right]^{2} x=0,
$$

where $\gamma$ is some effective damping and $\epsilon_{-}^{\mathrm{NP}-\mathrm{SP}}(t) \equiv$ $\epsilon_{-}^{\mathrm{NP}-\mathrm{SP}}\left(\eta_{p}(t)\right)$. The parametric resonance condition is determined by $\epsilon_{-}^{\mathrm{NP}-\mathrm{SP}}(t=0) / \omega=n / 2, n \in \mathbb{N}_{0}$ whereas the structure of the instability lobes is determined by the detailed form of $\left(\epsilon_{-}^{\mathrm{NP}-\mathrm{SP}}\right)$. The many-body resonance periods in our 


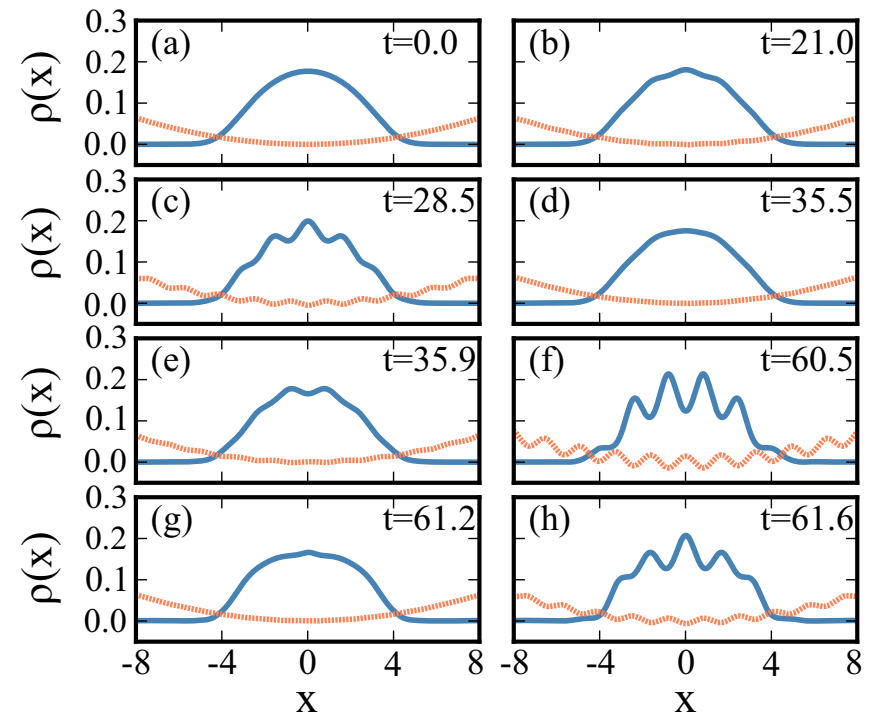

FIG. 3. Snapshots of the time evolution of the atomic density (solid blue curves) and of the effective potential (dashed orange curve) in the many-body parametric resonance phase. The density is normalized to 1 while the units of time and length are $0.63 \mathrm{~ms}$ and $0.48 \mu \mathrm{m}$, respectively. The condensate was driven starting from the (static) normal phase with fixed parameters $\eta_{p}^{0, \mathrm{NP}}=2 \pi \times 3.28 \mathrm{kHz}$, $\Delta_{c}=-2 \pi \times 10.08 \mathrm{MHz}$. One can clearly appreciate the superlattice switching between odd and even configurations. The driving from the SP leads to a qualitatively similar superlattice switching and is therefore not shown. All quantities shown are dimensionless; see Appendix $\mathrm{C}$ for further discussion.

simulations are in good agreement with this simple resonance condition for $n=1$ when the system is driven starting from the NP or the SP. The resulting lobes are the first instability lobes and the complex lobe shape in the SP case is qualitatively captured by Eq. (3). Higher values of the cavity dissipation $\kappa$ were found to smoothen the shape of the MaPaReP lobe (cf. Sec. III D).

\section{Time evolution of the atomic density}

Insights into the nature of the different phases can be gained by an analysis of the time-evolved density $\rho(x, t)$ and the effective one-body potential $V(x, t)$ seen by the atoms [see Figs. 1(c) and 3].

In the NP, the trapped BEC has a Gaussian profile $\Theta(t) \approx 0$ and $\rho(x, t)$ shows minimal changes as a function of time. The atomic density profile then looks as in Fig. 3(a).

In the SP, the atoms occupy the sites of the even or the odd lattice and $\Theta$ shows an oscillatory behavior with nonzero mean [cf. light blue line in Fig. 4(b)]. Exemplary density profiles for this phase are shown in Figs. 3(f) and 3(h). The choice of which superlattice is occupied can be seen as a manifestation of spontaneous symmetry breaking. Throughout the entire duration of the modulated plateau, the condensate does not leave the initially "chosen" symmetry subspace and the only dynamical feature observed is a deepening of the density minima with increasing pump power. Upon ramping down the laser pump, the condensate exhibits a quasi-irregular motion,
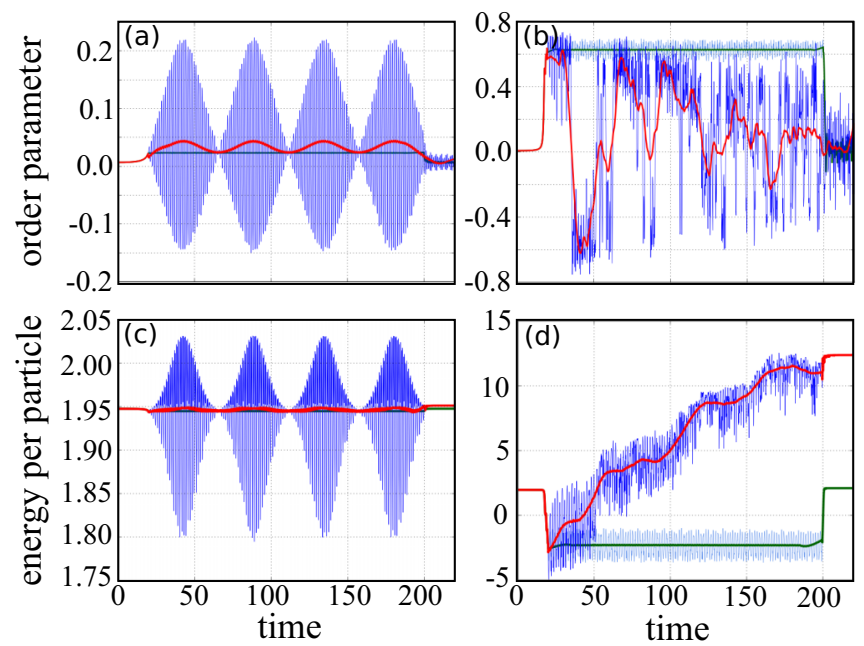

FIG. 4. Time evolution of the order parameter and energy for a system driven from the NP (left panels) and SP (right panels). Thin lines represent the raw data, while running averages are marked as thick solid lines. Each panel features one time evolution in the modulated NP or SP and another one in the corresponding MaPaReP at higher periods. The driving parameters $\alpha$ and $T$ are $(0.005,1.6)$ (light line) and $(0.05,1.35)$ (dark line) for the left panels, and $(0.04,1.6)$ (light line) and $(0.096,1.6)$ (dark line) for the right panels. In the driven NP we note that the amplitude of the oscillations in the raw energy curve is very small and hence they are covered by their running average (thick dark line). All quantities shown are dimensionless; see Appendix C.

signaling that it cannot simply go back to the initial Gaussianlike configuration.

In the modulated plateau region, cf. Fig. 1, the atoms remain in the lattice configuration chosen by the atoms before the pump modulation was turned on. In the MaPaReP, however, the atoms and their potential systematically oscillate between the even and the odd lattice configurations always passing through a Gaussian-like distribution [see Figs. 1(c) and $1(\mathrm{~d})$ and $3(\mathrm{c})-3(\mathrm{~h})$ ], hinting at a complex dynamical particle reconfiguration. The superlattice switching is induced by a dynamical change in the effective potential, which oscillates between an odd and an even configuration [compare, for example, Figs. 3(f) and 3(h)]. We see that the lattice contribution to the effective potential seen by the atoms goes to zero at the point where the atoms transition between the even and the odd lattice.

As expected from the general solutions of Mathieu-like equations $[39,40]$, both the density and the one-body potential oscillate in time not at the underlying driving frequency [see Fig. 1(c)], but rather aperiodically. As time progresses, the density peaks in the inequivalent superlattices gradually become more pronounced [Figs. 3(f)-3(h)], concomitant with an increase in energy, signaling that the condensate is heating up.

\section{Stability of many-body phases and heating characteristics}

We now discuss the stability of the different phases to heating, which is endemic to periodically driven interacting systems. In the NP and SP, the energies per particle oscillate 
(aperiodically) in time, but their time averages stay constant [see Figs. 4(c) and 4(d)]. Earlier works on the closed undriven Dicke model $[38,41]$ have shown the integrable nature of the NP and the ergodic nature of the SP. The lack of heating when modulating the NP is consistent with this integrability. This hints towards the existence of a generalized Gibbs ensemble $[42,43]$ describing the modulated NP. The fact that the modulated SP does not heat up at experimentally relevant time scales does not preclude heating and thermalization at much longer times not accessed in the simulation.

In the MaPaReP, the system tends to heat up irrespective of whether it originated from the NP or the SP. The heating across the entire phase is illustrated by the colored tiling of the phase diagrams in Fig. 2. Remarkably, the MaPaReP obtained from the NP (upper panel of Fig. 2) has minimal heating as compared to the MaPaReP obtained from the SP; it displays prethermalizationlike plateaus where the average energy is approximately constant [see thick solid curve in Fig. 4(c)] and $\Theta$ shows smooth oscillatory behavior. As the amplitude is gradually increased, the width of the plateaus shrinks and the condensate thermalizes more quickly. This rather stable behavior of the MaPaReP should make it easy to observe experimentally. The time scale over which the system absorbs energy depends crucially on the static pump rate $\eta_{p}^{0}$, the amplitude $\alpha$, and the period $T$.

The MaPaReP, when obtained from the SP, shows a much faster thermalization (increase of about $500 \%$ in the considered time interval) to a trivial high-temperature state [see Fig. 4(d)]. The corresponding order parameter $\Theta$ shows the even to odd lattice reconfigurations, but is increasingly noisy. The particularly high heating rate could stem from the underlying ergodic nature of the superradiant phase $[38,41]$. The jump from the nonheating (SP) to the heating behavior (MaPaReP) is sharp, suggesting a first-order phase transition. We find that the energy in the MaPaReP averaged over a period initially increases linearly with time despite the cavity dissipation.

We have also explored the robustness of our results to parameter changes in the system. We have investigated the stability of parametric resonance lobes with respect to sizable changes in the dissipation coefficient $\kappa$. We have simulated $N=1000$ interacting bosons in a cavity with the same parameters but different values of $\kappa$. The results are shown in Fig. 5. Besides some slight deformations in the boundaries of the Arnold tongue, we found no other significant change in the shape of the lobe, indicating that the many-body resonance is stable with respect to changes in the dissipation.

Similarly, different values of the cavity-atom coupling $g_{0}$ only affect the resonance condition of the instability lobes [17], shifting them vertically, but do not qualitatively change their physical picture. Additionally, the qualitative features of the heating diagrams are found to be insensitive to changes in the value of the atom contact interaction $U$ for experimentally relevant ranges, i.e., weakly interacting Bose gases, both for $M=1$ and beyond.

To summarize, heating profiles of the dissipative BECcavity system show an intriguing parametrically induced crossover between an effective integrability (where the system does not heat up) and nonintegrability (where the system heats up). We also reiterate that cavity fluctuations are not

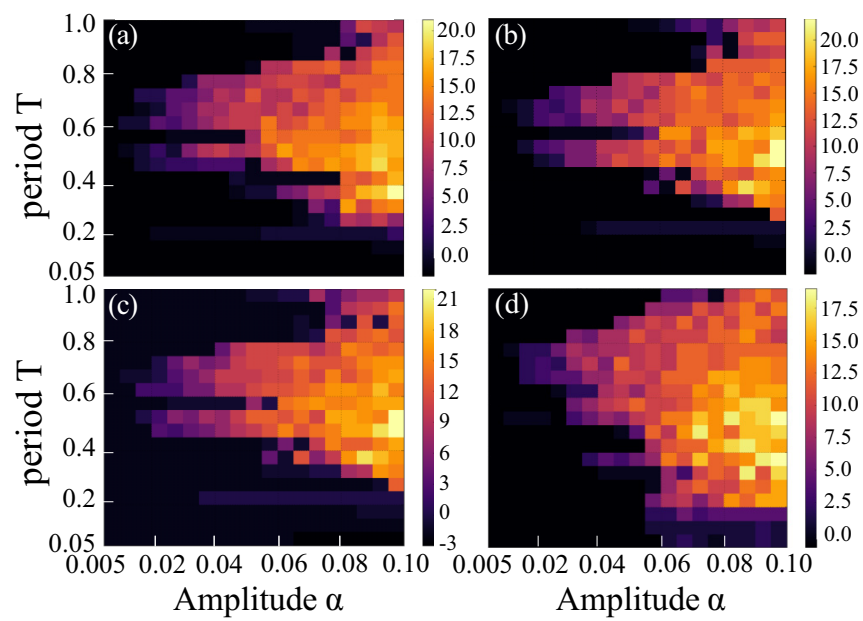

FIG. 5. Comparison of the parametric resonance (Arnold tongue) from the SP for different dissipation coefficients: (a) $\kappa=2 \pi \times$ 1.3 $\mathrm{MHz}$ (value used for the simulations presented in the main text), (b) $\kappa=2 \pi \times 0.13 \mathrm{MHz}$, (c) $\kappa=2 \pi \times 0.33 \mathrm{MHz}$, (d) $\kappa=2 \pi \times$ 2.6 MHz. All quantities shown are dimensionless; see Appendix C for further discussion.

retained in our analysis. This could prevent the activation of additional channels for cooling and could affect the overall heating behavior. The intriguing lack of heating in the SP and the general question of thermalization of the parametrically driven system merits further analysis, which is beyond the scope of the work presented here.

\section{E. Limitations of the two-level description in the MaPaReP}

In this section, we investigate the validity of the two-level picture in momentum space for the many-body parametric resonance phase $(\mathrm{MaPaReP})$. In the regimes where the twolevel description is valid the problem can be mapped to an effective parametrically driven Dicke model. A breakdown of the two-level description thus implies the appearance of physics beyond that of the Dicke model.

In both the normal and superradiant phases, the underlying physics is well approximated by a truncation of the description of the atoms to only contain the two lowest momentum states $[17,28,44]$. In the NP the momentum density $\rho(k, t)$ displays a single peak at $k=0$, while in the SP it shows two additional peaks at $k= \pm k_{c}$, where $k_{c} \approx 3.8$ is the quasimomentum of the spontaneously generated periodic potential.

While the two-level picture remains approximately valid for many points in the MaPaReP, the situation changes for certain thermalizing regions, where higher $k$ modes appear and the two-level picture breaks down. As presented in Fig. 6, where we plot the time evolution of $\rho(k, t)$ for times where the pump power is modulated in the MaPaReP [cf. Fig. 1(b)], the heating in the MaPaReP is concomitant with an increasing population of other momentum states beyond the integrable subspace of the $\pm k$ states [35], such as $\pm k_{c} / 2$ and $\pm 3 k_{c} / 2$, which are not included in the effective Dicke model description. Before the periodic modulation starts the system is undriven and in the SP with parameters $\eta_{p}^{0, S P}=2 \pi \times 4.54 \mathrm{kHz}$ and $\Delta_{c}=-2 \pi \times 10.08 \mathrm{MHz}$. Shortly after the laser modulation starts, apart from the large peak at $k=0$ corresponding 


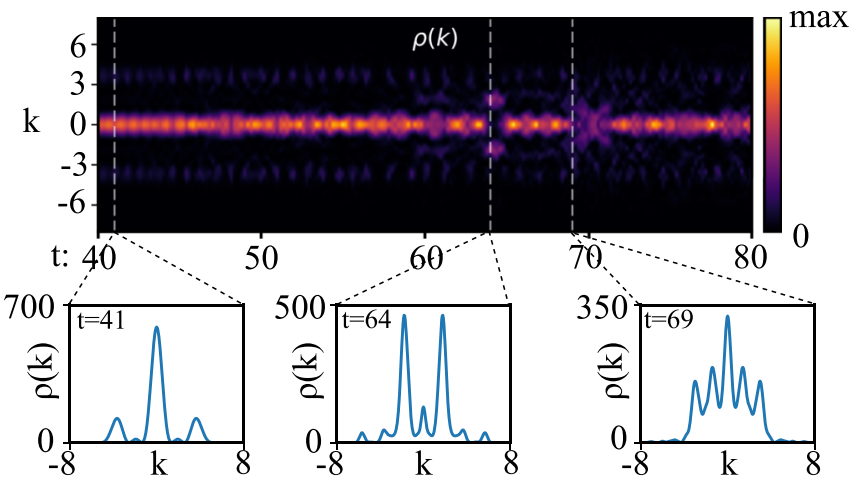

FIG. 6. Breakdown of the two-level picture in momentum space. Shown is the time evolution of the momentum density $\rho(k, t)$ for times where the pump power is modulated in the many-body parametric resonance phase. The condensate was driven starting from the (static) superradiant phase with fixed parameters $\eta_{p}^{0, \mathrm{SP}}=$ $2 \pi \times 4.54 \mathrm{kHz}$ and $\Delta_{c}=-2 \pi \times 10.08 \mathrm{MHz}$. Snapshots for three different representative times $(t=41,64,69)$ are shown. The time evolution is characterized by the emergence of peaks around multiples of $\pm k_{c} / 2$ and later at arbitrary positions, signaling a selfinterference of the condensate. All quantities shown are dimensionless; see Appendix C for further discussion.

to a flat density, $\rho(k, t)$ shows two sizable side peaks at $k= \pm k_{c}$ and emerging peaks at $k= \pm k_{c} / 2$ (see Fig. 6, left panel). At a later time $\rho(k, t)$ displays sizable peaks around $k=0, \pm k_{c} / 2$ and smaller peaks at $k= \pm k_{c}, \pm 3 k_{c} / 2$ (see Fig. 6, middle panel). Even later, $\rho(k, t)$ develops additional peaks at momenta which are not integer multiples of $\pm k_{c} / 2$, signaling a self-interference of the condensate. The increasing population of states beyond momenta $\pm k_{c}$ clearly indicates the inadequacy of the two-level description and hence the limitation of the mapping to the Dicke model in the MaPaReP.

\section{CONCLUSIONS}

We have investigated the full time evolution of a BEC coupled to a dissipative high-finesse optical cavity subject to a time-dependent transverse pumping laser power. We show that in addition to the static normal and superradiant phases, parametric driving leads to the formation of a new many-body parametric resonance phase $(\mathrm{MaPaReP})$ where the atoms switch quasiperiodically between the even- and odd-symmetric configurations. Such oscillations, which are explicitly forbidden in the undriven system, herald physics beyond the Dicke model picture. The boundaries of this dynamical phase are delineated by Arnold instability lobes. The driven NP and SP are resistant to heating-possibly pointing towards the existence of a generalized Gibbs ensemble and/or interesting prethermalization regimes. The MaPaReP, instead, shows prethermalization and eventually thermalizes fully despite the presence of dissipation. Possible future directions of research include investigations of the nature of the MaPaReP phase transition, its heating characteristics, analyses of the correlation functions within the condensate, probing the effect of fluctuations beyond mean field, and the inclusion of additional optical potential landscapes to compete with the
MaPaReP. An important outlook would be the generalization of such switching phases to other symmetry classes.

\section{ACKNOWLEDGMENTS}

The authors gratefully acknowledge C. Bruder, T. Donner, and O. Zilberberg for helpful discussions and comments on our work. We acknowledge financial support from G. Anderheggen. A.U.J.L. acknowledges financial support by the Swiss SNF and the NCCR Quantum Science and Technology and by the Austrian Science Foundation (FWF) under Grant No. F65 (SFB "Complexity in PDEs") and the Wiener Wissenschafts und TechnologieFonds (WWTF) Project No. MA16-066 ("SEQUEX"). Computation time on the Hazel Hen cluster of the HLRS in Stuttgart is gratefully acknowledged.

P.M. and L.P. contributed equally to this work.

\section{APPENDIX}

The results of the article are based on the numerical method and the Hamiltonian described in Secs. A and B of the appendixes. In Sec. C we discuss the parameters used for the computations, while in Sec. D we present the mapping of the driven Bose-Einstein condensate in a cavity to the driven Dicke model.

\section{APPENDIX A: NUMERICAL METHOD: MCTDH-X}

The multiconfigurational time-dependent Hartree method for indistinguishable particles (MCTDH-X), which we use to compute the time-evolution of the many-body wave function, is based on the following ansatz:

$$
|\Psi(t)\rangle=\sum_{\mathbf{n}} C_{\mathbf{n}}(t)|\mathbf{n}, t\rangle
$$

$|\Psi(t)\rangle$ is written in the basis of symmetrized states (permanents) $|\mathbf{n}, t\rangle$, which consist of products of $N$ particles in $M$ single-particle functions (orbitals) $\Phi_{i}(\mathbf{r}, t)$, with $i=$ $1, \ldots, M$. The number of orbitals $M$ can be chosen at will and in the limit of $M \rightarrow \infty$ the expansion in Eq. (A1) becomes exact. Note that both the weights $C_{\mathbf{n}}(t)$ and the permanents $|\mathbf{n}, t\rangle$ appearing in Eq. (A1) are explicitly time dependent. We can express the configurations $|\mathbf{n}, t\rangle$ as

$$
|\mathbf{n}, t\rangle=\prod_{i=1}^{M}\left[\frac{\left(\hat{b}_{i}^{\dagger}(t)\right)^{n_{i}}}{\sqrt{n_{i} !}}\right]|\mathrm{vac}\rangle,
$$

where the operator $\hat{b}_{i}^{\dagger}(t)$ creates a boson in the $i$ th singleparticle state $\Phi_{i}(\mathbf{r}, t)$ and $|\mathrm{vac}\rangle$ is the vacuum.

Using the time-dependent variational principle [45] the time-dependent many-body Schrödinger equation with the ansatz in Eq. (A1), one finds the following time evolution of the coefficients $\left\{C_{\mathbf{n}}(t)\right\}$ and orbitals $\left\{\Phi_{i}(\mathbf{r}, t)\right\}_{i=1}^{M}$ :

$$
i \partial_{t}\left|\Phi_{j}\right\rangle=\hat{P}\left[\hat{h}\left|\Phi_{j}\right\rangle+\lambda_{0} \sum_{k, s, q, l=1}^{M}\{\boldsymbol{\rho}\}_{j k}^{-1} \rho_{k s q l} \hat{W}_{s l}\left|\Phi_{q}\right\rangle\right],
$$

These are the coupled equations of motion of the MCTDHX [34,36] method. Here, $\rho_{k q}=\left\langle\Psi\left|\hat{b}_{k}^{\dagger} \hat{b}_{q}\right| \Psi\right\rangle$ and $\rho_{k q s l}=$ 
$\left\langle\Psi\left|\hat{b}_{k}^{\dagger} \hat{b}_{s}^{\dagger} \hat{b}_{l} \hat{b}_{q}\right| \Psi\right\rangle$ are the matrix elements of the reduced onebody and two-body density matrix. The one-body Hamiltonian is given by $\hat{h}$ (see definition in Sec. B) and $\hat{W}_{s l}(\mathbf{r})=$ $\int d \mathbf{r}^{\prime} \Phi_{s}^{*}\left(\mathbf{r}^{\prime}, t\right) \hat{W}\left(\mathbf{r}, \mathbf{r}^{\prime}, t\right) \Phi_{l}\left(\mathbf{r}^{\prime}, t\right)$ are local time-dependent interaction potentials, in which $\hat{W}\left(\mathbf{r}, \mathbf{r}^{\prime}, t\right)$ is the two-body interparticle interaction, and $\hat{P}$ is a projection operator onto the subspace orthogonal to that spanned by the orbitals $\left\{\Phi_{i}(\mathbf{r}, t)\right\}$. All coefficients $\left\{C_{\mathbf{n}}(t)\right\}$ are collected into the vector $\mathcal{C}(t)$ and $\mathcal{H}_{\mathbf{n}, \mathbf{n}^{\prime}}=\left\langle\mathbf{n}^{\prime}, t|\hat{H}| \mathbf{n}, t\right\rangle$ is the Hamiltonian represented in the chosen many-body basis. For the details of the derivation of Eqs. (A3) and (A4), see Refs. [34,36].

\section{APPENDIX B: HAMILTONIAN OF A MANY-BODY SYSTEM COUPLED TO A SINGLE-MODE CAVITY}

When situated inside an optical cavity, the one-body Hamiltonian $\hat{h}$ [cf. Eq. (A3)] has the form $\hat{h}=\hat{T}+V=\hat{T}+$ $V_{\text {trap }}+V_{\text {cavity }}$, where $\hat{T}$ is the kinetic energy and $V_{\text {trap }}$ is the external potential. The potential $V_{\text {cavity }}$ modifies the one-body Hamiltonian $\hat{h}$ in a time-dependent way: the atoms interact with the cavity field $a(t)$.

Since our numerical results in the main text are for a onedimensional system, we use the coordinate $x$ instead of $\mathbf{r}$ in the following.

The cavity's field generates a potential $U(x)$ which contributes to the one-body potential $V_{\text {cavity }}$,

$$
U(x)=\frac{g_{0}^{2}}{\Delta_{a}} \cos ^{2}(k x) .
$$

In addition, photons are scattered from the pump into the cavity field by the atoms, resulting in a cavity photon source term of the form

$$
\eta(x, t)=\frac{g_{0} \Omega_{p}(t)}{\Delta_{a}} \cos (k x) .
$$

Here $g_{0}$ is the atom-cavity coupling, $\Delta_{a}$ is the atomic detuning, and $\Omega_{p}$ is the pump Rabi frequency, which depends on the pump laser power and in our case is explicitly time dependent. The combined one-body potential entering the many-body Hamiltonian of the combined system then reads

$$
\begin{aligned}
V(x, t)= & V_{\text {trap }}(x)+V_{\text {cavity }} \\
= & V_{\text {trap }}(x)+V_{0}(t)+|a(t)|^{2} U(x) \\
& +\left[a(t)+a^{*}(t)\right] \eta(x, t) .
\end{aligned}
$$

Here, $V_{\text {trap }}(x, t)=\frac{1}{2} \omega_{x}^{2} x^{2}$ is the external trapping potential for the bosons, without the cavity, and $V_{0}(t)=\frac{\Omega_{p}^{2}(t)}{\Delta_{a}}$ is the potential created by the pump field.

The equation of motion of the cavity field $a(t)$ is given by

$$
\begin{aligned}
i \partial_{t} a(t)= & {\left[-\Delta_{c}+\sum_{k, q=1}^{M}\left(\rho_{k q}(t) U_{k q}\right)-i \kappa\right] a(t) } \\
& +\sum_{k, q=1}^{M}\left[\rho_{k q}(t) \eta_{k q}(t)\right],
\end{aligned}
$$

where we have introduced the matrix elements $U_{k q}(t) \equiv$ $\left\langle\Phi_{k}|U(x)| \Phi_{q}\right\rangle$ and $\eta_{k q}(t) \equiv\left\langle\Phi_{k}|\eta(x, t)| \Phi_{q}\right\rangle$ using the orbitals $\Phi_{i}$ (see Sec. A) and $\Delta_{c}$ is the cavity detuning. The complex damping term $-i \kappa$ has been introduced in order to deal with photon losses using the settled set of equations of motion; cf. (A3), (A4), (B3).

\section{APPENDIX C: PARAMETERS}

We have simulated the full-time evolution of the BECcavity system with $N=1000$ atoms in a cavity driven by a transverse pump laser; cf. Fig. 1 of the main text. The dimensionless parameters used throughout the work are computed as follows. First we fix the length scale $L=0.48 \mu \mathrm{m}$. The scale of energy for the specific choice of ${ }^{87} \mathrm{Rb}$ atoms is $\hbar^{2} / 2 m L^{2} \approx$ $1.67 \times 10^{-31} \mathrm{~J}$. Similarly, the scale of time is $2 m L^{2} / \hbar \approx$ $0.63 \mathrm{~ms}$. In our computations the atoms have been chosen to interact weakly with each other with an interaction strength of $\lambda_{0} \approx 0.01$ [cf. Eq. (A3)]. In experiments $\lambda_{0}$ can be chosen by tuning the frequency of the transverse confining harmonic potential $\omega_{\perp}$. The one-dimensional scattering strength $\lambda_{0}$ is related to the three-dimensional scattering length $a$ through $\lambda_{0}=4 L m \omega_{\perp} a / \hbar[26]$. Using the listed parameters, and the fact that $a \approx 100 a_{0}$ for ${ }^{87} \mathrm{Rb}$, one obtains $\omega_{\perp} \approx 718 \mathrm{~Hz}$. The atom-cavity coupling $U_{0} \approx 338.22 \mathrm{~Hz}$, the cavity resonance frequency $\omega_{k} \approx 2 \pi \times 382.41 \mathrm{THz}$, and the loss rate $\kappa \approx$ $2 \pi \times 1.3 \mathrm{MHz}$ have been chosen in accordance with experimental values [17].

By measuring the order parameter $\Theta \equiv\langle\psi|\cos k x| \psi\rangle$ for this set of parameters we have first obtained the static phase diagram (see inset of Fig. 7) as a function of pump rate $\eta_{p}$ and detuning $\Delta_{c}$. Subsequently, we have fixed the detuning at a value $\Delta_{c}=-2 \pi \times 10.08 \mathrm{MHz}$ and chosen two representative pump rates $\eta_{p}^{\mathrm{NP}}=2 \pi \times 3.28 \mathrm{kHz}$ and $\eta_{p}^{\mathrm{SP}}=2 \pi \times$ $4.79 \mathrm{kHz}$ to investigate the effect of a sinusoidal modulation of the drive in the plateau region. We have again extracted the order parameter $\Theta$ and then obtained the phase diagram of the driven BEC-cavity system as a function of period $T$ and intensity $\alpha$ of the drive (see Fig. 7). A point in the $(\alpha, T)$ phase diagram is classified as belonging to the MaPaReP if its order parameter oscillates in time between positive and negative values while its long-time average is vanishingly small.

\section{APPENDIX D: MAPPING TO THE DRIVEN DICKE MODEL}

The Hamiltonian of the periodically driven Dicke model is given by $[22,23]$

$$
\mathcal{H}_{S}(t)=\omega_{c} \hat{a}^{\dagger} \hat{a}+\omega_{a} \hat{J}_{z}+\frac{\mu(t)}{\sqrt{N}}\left(\hat{a}^{\dagger}+\hat{a}\right)\left(\hat{J}_{+}+\hat{J}_{-}\right),
$$

where $\omega_{c}$ is the cavity resonance frequency and $\omega_{a}$ is the level spacing of the two-level atoms. The operators $\hat{a}^{(\dagger)}$ are the standard annihilation or creation operators of the cavity field, while $\hat{J}_{z}, \hat{J}_{+}, \hat{J}_{-}$are the atomic collective operators. The atom-cavity coupling is time dependent and has the following explicit form: $\mu(t)=\mu_{0}+\alpha \cos (2 \Omega t)$. Using the standard Holstein-Primakoff transformation [38,46], and assuming the number of particles $N$ to be very large, allows us to write the Hamiltonian (D1) in terms of bosonic operators $\hat{d}^{(\dagger)}$ as

$$
H_{S}(t)=\omega_{c} a^{\dagger} a+\omega_{a} d^{\dagger} d+\mu(t)\left(a^{\dagger}+a\right)\left(d^{\dagger}+d\right) .
$$



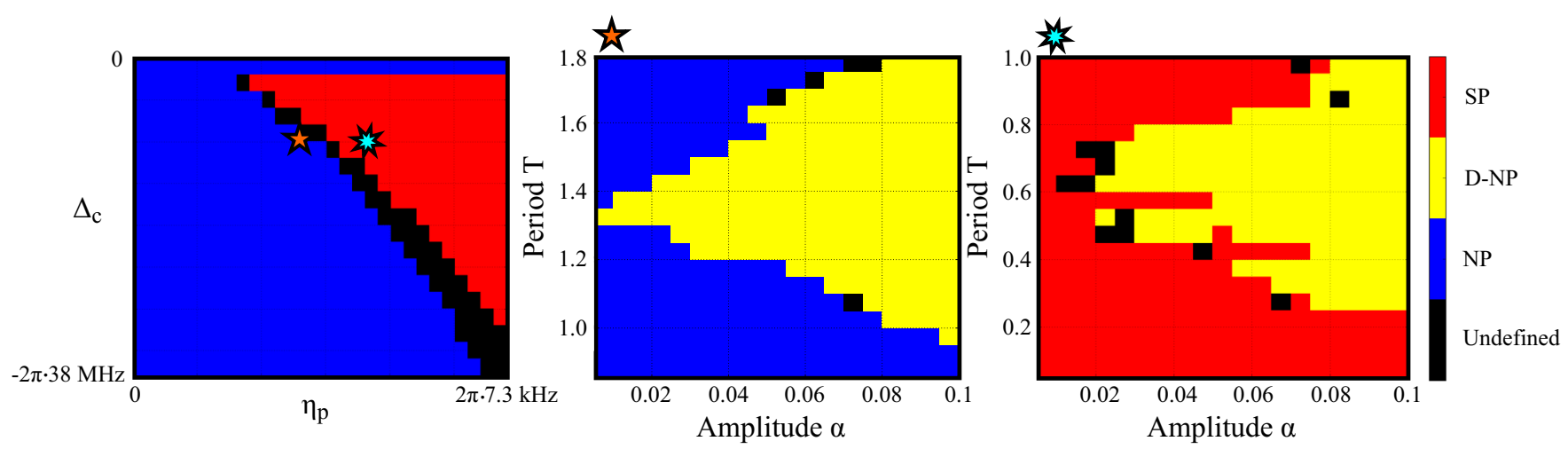

FIG. 7. Illustration of the many-body resonances (Arnold lobes) and the corresponding phase transition to the MaPaReP for driving from the NP and from the SP. The parameters that drive this phase transition are the amplitude $\alpha$ of the drive and its period $T$. The inset shows the static NP or SP phase diagram as a function of pump rate $\eta_{p}$ and detuning $\Delta_{c}$. All quantities shown are dimensionless; see Appendix C for further discussion.

The Hamiltonian (D2) can be decoupled through a normalmode transformation, leading to

$$
H_{S}(t)=\Omega_{1} c_{1}^{\dagger} c_{1}+\Omega_{2} c_{2}^{\dagger} c_{2}
$$

which is the Hamiltonian of two parametrically driven oscillators. The normal-mode (polariton) dispersions for the experimental case $\left(\omega_{c} \gg \omega_{a}\right)$ for the NP are given by

$$
\begin{aligned}
& \left(\Omega_{1}^{\mathrm{NP}}\right)^{2}=\omega_{c}^{2}+4 \mu^{2}(t) \frac{\omega_{a}}{\omega_{c}}, \\
& \left(\Omega_{2}^{\mathrm{NP}}\right)^{2}=\omega_{a}^{2}-4 \mu^{2}(t) \frac{\omega_{a}}{\omega_{c}},
\end{aligned}
$$

and for the SP by

$$
\begin{gathered}
\left(\Omega_{1}^{\mathrm{SP}}\right)^{2}=\omega_{c}^{2}-\omega_{a}^{2} \\
\left(\Omega_{2}^{\mathrm{SP}}\right)^{2}=16 \frac{\mu^{4}(t)}{\omega_{c}^{2}}-\omega_{a}^{2} .
\end{gathered}
$$

Even though the system we study is more complex than the simple driven Dicke model studied in Ref. [23], this mapping helps us to estimate the periods at which the parametric instabilities emerge (cf. main text).
[1] S. Ritter, F. Brennecke, K. Baumann, T. Donner, C. Guerlin, and T. Esslinger, Appl. Phys. B 95, 213 (2009).

[2] T. P. Purdy, D. W. C. Brooks, T. Botter, N. Brahms, Z.-Y. Ma, and D. M. Stamper-Kurn, Phys. Rev. Lett. 105, 133602 (2010).

[3] A. Eckardt and E. Anisimovas, New J. Phys. 17, 093039 (2015).

[4] F. Meinert, M. J. Mark, K. Lauber, A. J. Daley, and H.-C. Nägerl, Phys. Rev. Lett. 116, 205301 (2016).

[5] N. Goldman and J. Dalibard, Phys. Rev. X 4, 031027 (2014).

[6] L. Landau and E. Lifshitz, Mechanics (ButterworthHeinemann, Oxford, 1976).

[7] G. Casati, B. V. Chirikov, F. M. Izraelev, and J. Ford, in Stochastic Behavior in Classical and Quantum Hamiltonian Systems, Lecture Notes in Physics Vol. 93 (Springer, Berlin, 1979), pp. 334-352.

[8] S. Fishman, D. R. Grempel, and R. E. Prange, Phys. Rev. Lett. 49, 509 (1982).

[9] A. Lazarides, A. Das, and R. Moessner, Phys. Rev. E 90, 012110 (2014).

[10] L. D’Alessio and M. Rigol, Phys. Rev. X 4, 041048 (2014).

[11] P. Ponte, A. Chandran, Z. Papić, and D. A. Abanin, Ann. Phys. 353, 196 (2015).

[12] J. Keeling, L. M. Sieberer, E. Altman, L. Chen, S. Diehl, and J. Toner, in Universal Themes of Bose-Einstein Condensation, edited by N. P. Proukakis, D. W. Snoke, and P. B. Littlewood (Cambridge University Press, Cambridge, 2017), pp. 205-230.

[13] R. H. Dicke, Phys. Rev. 93, 99 (1954).
[14] K. Hepp and E. Lieb, Phys. Rev. A 8, 2517 (1973).

[15] P. Domokos and H. Ritsch, Phys. Rev. Lett. 89, 253003 (2002).

[16] F. Dimer, B. Estienne, A. S. Parkins, and H. J. Carmichael, Phys. Rev. A 75, 013804 (2007).

[17] K. Baumann, C. Guerlin, F. Brennecke, and T. Esslinger, Nature (London) 464, 1301 (2010).

[18] K. Baumann, R. Mottl, F. Brennecke, and T. Esslinger, Phys. Rev. Lett. 107, 140402 (2011).

[19] J. Klinder, H. Keßler, M. Wolke, L. Mathey, and A. Hemmerich, Proc. Natl. Acad. Sci. USA 112, 3290 (2015).

[20] M. R. Bakhtiari, A. Hemmerich, H. Ritsch, and M. Thorwart, Phys. Rev. Lett. 114, 123601 (2015).

[21] F. Brennecke, R. Mottl, K. Baumann, R. Landig, T. Donner, and T. Esslinger, Proc. Natl. Acad. Sci. USA 110, 11763 (2013)

[22] V. M. Bastidas, C. Emary, B. Regler, and T. Brandes, Phys. Rev. Lett. 108, 043003 (2012).

[23] R. Chitra and O. Zilberberg, Phys. Rev. A 92, 023815 (2015).

[24] D. Nagy, G. Szirmai, and P. Domokos, Eur. Phys. J. D 48, 127 (2008).

[25] K. Huang, Statistical Mechanics (Wiley, New York, 1987).

[26] M. Olshanii, Phys. Rev. Lett. 81, 938 (1998).

[27] C. J. Pethick and H. Smith, Bose-Einstein Condensation in Dilute Gases, 2nd ed. (Cambridge University Press, Cambridge, UK, 2008).

[28] D. Nagy, G. Kónya, G. Szirmai, and P. Domokos, Phys. Rev. Lett. 104, 130401 (2010). 
[29] G. Kurizki, I. E. Mazets, D. H. J. O'Dell, and W. P. Schleich, Int. J. Mod. Phys. 18, 961 (2004).

[30] D.-G. Lai, F. Zou, B.-P. Hou, Y.-F. Xiao, and J.-Q. Liao, Phys. Rev. A 98, 023860 (2018).

[31] A. U. J. Lode, M. C. Tsatsos, and E. Fasshauer, The Timedependent Multiconfigurational Hartree Method for Indistinguishable Particles Software, http://ultracold.org.

[32] E. Fasshauer and A. U. J. Lode, Phys. Rev. A 93, 033635 (2016).

[33] A. U. J. Lode, Phys. Rev. A 93, 063601 (2016).

[34] O. E. Alon, A. I. Streltsov, and L. S. Cederbaum, J. Chem. Phys. 127, 154103 (2007).

[35] A. U. J. Lode and C. Bruder, Phys. Rev. Lett. 118, 013603 (2017).

[36] O. E. Alon, A. I. Streltsov, and L. S. Cederbaum, Phys. Rev. A 77, 033613 (2008).

[37] A. I. Streltsov, O. E. Alon, and L. S. Cederbaum, Phys. Rev. Lett. 99, 030402 (2007).
[38] C. Emary and T. Brandes, Phys. Rev. E 67, 066203 (2003).

[39] W. Magnus and S. Winkler, Hill's Equation (Interscience Publishers, New York, 1966).

[40] N. McLachlan, Theory and Application of Mathieu Functions (Clarendon, Oxford, 1951).

[41] A. Altland and F. Haake, Phys. Rev. Lett. 108, 073601 (2012).

[42] T. Langen, T. Gasenzer, and J. Schmiedmayer, J. Stat. Mech. (2016) 064009.

[43] T. Langen, S. Erne, R. Geiger, B. Rauer, T. Schweigler, M. Kuhnert, W. Rohringer, I. E. Mazets, T. Gasenzer, and J. Schmiedmayer, Science 348, 207 (2015).

[44] F. Piazza, S. Philipp, and W. Zwerger, Ann. Phys. 339, 135 (2013).

[45] P. Kramer and M. Saraceno (eds.), Geometry of the TimeDependent Variational Principle in Quantum Mechanics (Springer, Heidelberg, 1981).

[46] T. Holstein and H. Primakoff, Phys. Rev. 58, 1098 (1940). 